\title{
ADOPT-Studie
}

\section{Längere Thromboseprophylaxe bei internistischen Patienten ohne Vorteil}

\begin{abstract}
Akut erkrankte internistischen Patienten haben ein erhöhtes Thromboembolierisiko über die Zeit des Krankhausaufenthaltes hinaus. Eine längere Thromboseprophylaxe kann jedoch nicht empfohlen werden. Erneut fand eine große Studie keinen deutlichen Nutzen dafür.
\end{abstract}

- Das Thromboembolie-Risiko sowohl von Patienten nach großen orthopädischen Operationen als auch von akut schwer erkrankten internistischen Patienten bleibt bis zu drei Monate lang erhöht. In beiden Patientenpopulationen hat sich eine pharmakologische Thromboseprophylaxe während des Krankenhausaufenthaltes als sehr wirksam und sicher erwiesen.

Bei chirurgischen Patienten wird von den Leitlinien darüber hinaus eine Fortführung der Thromboseprophylaxe zuhause während der Zeit der Immobilisation empfohlen. Ein solches Vorgehen reduziert das Risiko für symptomatische und asymptomatische Thromboembolien.

\section{Zwei Drittel aller Thromboembolien bei internistischen Patienten}

Bei akut erkrankten internistischen Patienten, bei denen über zwei Drittel aller Thromboembolien beobachtet werden, ist die Datenlage weniger eindeutig. $\mathrm{Ob}$ eine über den Krankenhausaufenthalt hinaus dauernde längere Thromboseprophylaxe sinnvoll ist, wurde bisher in den beiden Studien EXCLAIM mit Enoxaparin und MAGELLAN mit Rivaroxaban untersucht.

Beide fanden zwar signifikant weniger Thromboembolien unter einer verlängerten Prophylaxe. Doch der Vorteil wurde in beiden Fällen durch ein Plus an schweren Blutungen geschmälert oder wettgemacht.

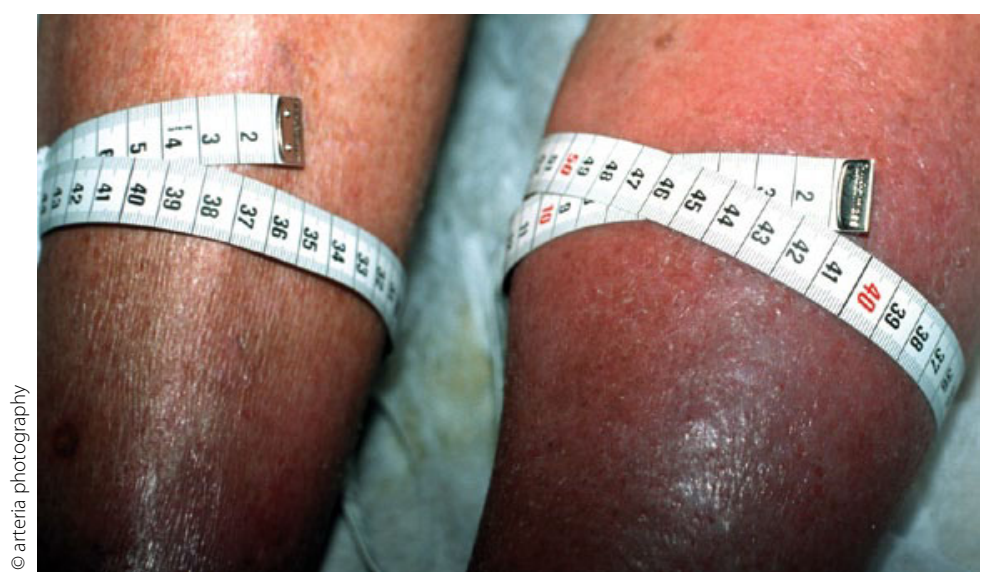

Venenthrombose am linken Unterschenkel.

\section{Die ADOPT-Studie}

Nun wurde mit der ADOPT-Studie eine dritte Doppelblindstudie mit internistischen Patienten publiziert. Verglichen wurden Enoxaparin $40 \mathrm{mg} / \mathrm{d}$ s.c. über im Schnitt 7 Tage mit dem Apixaban $2 x$ $2,5 \mathrm{mg} / \mathrm{d}$ oral über im Schnitt 25 Tage. Apixaban (Eliquis ${ }^{\circ}$ ) ist ein orales Antikoagulans, welches selektiv den Faktor Xa hemmt.

Die Studie bestätigt erneut, dass das Thromboserisiko dieser Patienten über den Zeitraum der Hospitalisation hinaus anhält. Allerdings fand sie keinen Vorteil für die längere Therapie: Innerhalb von 30 Tagen wurden bei $60 \mathrm{~Pa}$ tienten $(2,71 \%)$ der Apixaban-Gruppe sowie bei 70 Patienten $(3,06 \%)$ der Enoxaparin-Gruppe Thromboembolien diagnostiziert (primärer Endpunkt). Gleichzeitig traten mit 0,47\% (15 Patienten) in der Apixaban-Gruppe mehr schwere Blutungen auf als in der Enoxaparin-Gruppe (0,19\%, 6 Patienten).

\section{Studie underpowered}

Ein wesentlicher Grund für das negative Studienergebnis war nach Ansicht von Studienautor Dr. Samuel Goldhaber vom Brigham und Women's Hospital in Boston die hohe Drop-Out-Rate: Von den randomisierten 6528 Patienten konnten nur 4495 Patienten ausgewertet werden, weil in jeder Gruppe jeweils 1000 der alten und kranken Patienten nicht mehr für die Kompressions-Ultraschalluntersuchungen zur Verfügung standen, mit denen am Tag der Entlassung sowie nach 30 Tagen nach Thromboembolien gefahndet wurde. Dadurch war die Studie nicht mehr groß genug, um einen Vorteil der längeren Thromboseprophylaxe aufzuzeigen.

Die Studie hinterlässt die Experten ratlos: Obwohl das Thromboserisiko dieser internistischen Patienten nach der Krankenhausentlassung deutlich erhöht bleibt, kann eine verlängerte Thromboseprophylaxe aufgrund der vorliegenden drei Studien nicht empfohlen werden. Wir müssen herausfinden, welche Patienten ein hohes Risiko aufweisen, resümierte die Diskutantin der Studie, Dr. Mary Cushman aus Burlington/Vermont.

DR. MED. DIRK EINECKE .

\footnotetext{
- Quelle: American Heart Association, Scientific Sessions 2011, Late Breaking Clinical Trials, Session 2, Orlando, November 2011; Goldhaber S.Z. et al. Apixaban versus Enoxaparin for Thromboprophylaxis in Medically III Patients. N Engl J Med 2011; DOI: 10.1056/ NEJMoa1110899
} 